\title{
A Test of New Large Aperture Photodetectors in a Water Cherenkov Detector
}

\author{
Yasuhiro NISHIMURA* ${ }^{* \dagger}$ \\ Research Center for Cosmic Neutrinos, Institute for Cosmic Ray Research, The University of \\ Tokyo \\ E-mail: vnisieicrr.u-tokyo.ac.jp
}

Yuji OKAJIMA ${ }^{a}$, Miao JIANG $^{b}$, Daisuke FUKUDA ${ }^{c}$, Ryosuke AKUTSU, Yusuke SUDA $^{d}$, Seiko HIROTA ${ }^{b}$, Shoei NAKAYAMA ${ }^{e}$, Masato SHIOZAWA ${ }^{e}$, Masashi YOKOYAMA $^{d}$, Yoshinari HAYATO ${ }^{e}$, Hidekazu TANAKA ${ }^{e}$, Masayuki NAKAHATA $^{e}$, Tsuyoshi NAKAYA $^{b}$, Masahiro KUZE ${ }^{a}$, Yusuke $\mathrm{KOSHIO}^{c}$, Akimichi TAKETA $^{f}$

${ }^{a}$ Department of Physics, Tokyo Institute of Technology

${ }^{b}$ Department of Physics, Kyoto University

${ }^{c}$ Department of Physics, Okayama University

${ }^{d}$ Department of Physics, The University of Tokyo

${ }^{e}$ Kamioka Observatory, Institute for Cosmic Ray Research, The University of Tokyo

${ }^{f}$ Earthquake Research Institute, The University of Tokyo

\begin{abstract}
Several new photodetectors with high resolution and efficiency have been developed for a one megaton water Cherenkov detector, Hyper-Kamiokande, planned in Japan. A reliable photodetector is essential for such a long-term experiment aiming at the neutrino physics and the nucleon decay search. In 2013, a first proof test started using a 200 ton water Cherenkov detector in order to evaluate the feasibility of new photodetector techniques for Hyper-Kamiokande: high quantum efficiency (QE), a hybrid photodetector (HPD) and a new photomultiplier tube (PMT) with a box-and-line dynode. Eight HPDs with a $20 \mathrm{~cm}$ diameter were installed to the 200-ton water tank in advance of a $50 \mathrm{~cm}$ HPD. The QE of $50 \mathrm{~cm}$ Venetian-blind-dynode PMT used in SuperKamiokande was improved to $30 \%$ from $22 \%$. Five of such high-QE PMTs were attached as well in 2013. Three $50 \mathrm{~cm}$ high-QE box-and-line PMTs joined in 2014 and showed high detection performance. In the 200-ton tank, in total 240 photodetectors including the $50 \mathrm{~cm}$ conventional Super-Kamiokande PMTs have been tested over a year. Sufficient performance to detect the single photoelectron was confirmed in the tank; 40-45\% charge resolution in sigma and $5.1 \mathrm{nsec}$ timing resolution at FWHM for box-and-line PMTs. New photodetectors were monitored over a year, and a stability of gain is confirmed in $2 \%$ level. The test allows to select the best candidate photodetector for Hyper-Kamiokande, with coming $50 \mathrm{~cm}$ high-QE HPDs.
\end{abstract}

International Conference on New Photo-detectors

6-9 July 2015

Moscow, Troitsk, Russia

\footnotetext{
*Speaker.

${ }^{\dagger}$ Acknowledgments: JSPS KAKENHI Grant Number 25707016, EGADS working group
} 


\section{Introduction}

Hyper-Kamiokande (Hyper-K) is a next generation water Cherenkov detector planned in Kamioka, Japan [四]. It is an upgrade of Super-Kamiokande (Super-K), currently running to study various physics topics of neutrinos and nucleon decays [[]].

With dimensions $48(\mathrm{~W}) \times 54(\mathrm{H}) \times 250(\mathrm{~L}) \mathrm{m}^{3}$ for each tank, the total (fiducial) volume of Hyper-K two tanks will be 1.0 (0.56) million metric tons, which will be 20 (25) times larger than that of Super-K. The Hyper-K detector requires 99,000 photodetectors with a $50 \mathrm{~cm}$ diameter for a detection volume, and 25,000 with $20 \mathrm{~cm}$ for an outer veto layer to reject cosmic-ray muons.

In order to improve conventional photomultiplier tubes (PMTs) used in Super-K, $50 \mathrm{~cm}$ R3600 (Super-K PMT [B]]) and $20 \mathrm{~cm}$ R5912 provided by Hamamatsu Photonics K.K. (Hamamatsu), we developed new photodetectors. To prove a long and safe operation of new photodetectors, a proof test in a water Cherenkov detector has started. It would help determining the best photodetector for Hyper-K.

\section{Photodetectors}

Two types of new large-aperture photodetectors, manufactured by Hamamatsu, are developed. One is the PMT with a box-and-line dynode (Box\&Line PMT) for a good charge and time resolution. It is also equipped with a photocathode with a high quantum efficiency (QE). A hybrid photodetector (HPD) is another new photodetector equipped with an avalanche diode, which would bring an excellent charge and time resolution with a low production cost.

A small $20 \mathrm{~cm}$ HPD was developed prior to $50 \mathrm{~cm}$ one in order to establish the technology of the HPD for manufacturing. Also high QE cathode is firstly applied on the Super-K PMT as a part of training in order to optimize the high QE technique with minimizing the dark noise. The $50 \mathrm{~cm}$ high-QE HPD and Box\&Line PMT are possible candidates for Hyper-K. These initial prototypes are currently under the proof test and described in this section.

\section{$2.120 \mathrm{~cm}$ Hybrid Photo-Detector}

Hybrid photodetector, HPD, uses a different amplification system of the avalanche diode (AD) compared with the dynode for the PMT. A high voltage bias around eight $\mathrm{kV}$ is required to focus photoelectrons into the small AD surface area of $5 \mathrm{~mm}$ diameter from the $20 \mathrm{~cm}$ large aperture. It is high compared with two kilovolts applied to the Super-K PMT, accordingly the high voltage cable and connection are concern to be used in water. A dedicated compact high voltage power supply was developed and enveloped in the HPD waterproof module, as well as a preamplifier. Thus, only ten volts are necessary to operate the HPD with another control lines (up to $5 \mathrm{~V}$ ) of the module.

Another bias voltage of hundreds volts is required for the $\mathrm{AD}$, which provides another amplification gain $(50-100)$ after a bombardment gain $(\sim 1,600)$ by eight kilovolts. A gain of $10^{7-8}$, which is comparable with that of the PMT, is realized with $10^{5}$ of a total HPD gain and the following preamplifier.

Figures $\square$ and $\square$ show the layout and preamplifier system in the $20 \mathrm{~cm}$ HPD. The HPD demands control cables consisting of an enable switch, analog control of two high voltages, and $+10 \mathrm{~V}$ power, thus the waterproof cable complex with a coaxial signal cable was also prepared. 


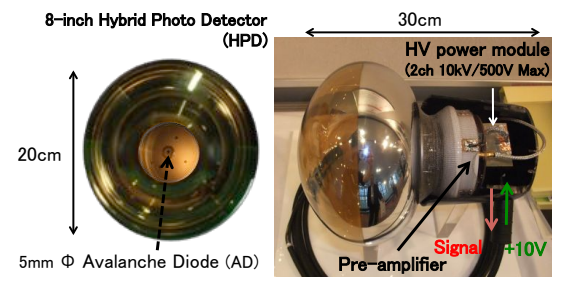

Figure 1: The $20 \mathrm{~cm}$ HPD (normal QE) and end-cap module. It is equipped with a high voltage module and preamplifier in a waterproof end cap.

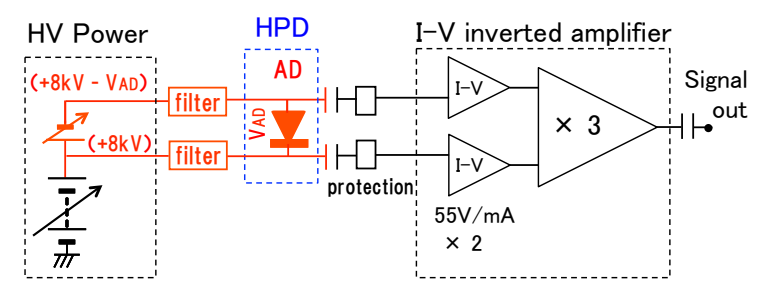

Figure 2: Preamplifier of the $20 \mathrm{~cm}$ HPD.

The HPD acquires a good charge and time resolutions by the high bombardment gain at the first stage than that of the Super-K PMT $(\sim 15)$. Also the high voltage allows a fast response and high magnetic field tolerance. However, the fast HPD signal is smeared in the currently adopted preamplifier and then the waveform becomes comparable with that of PMT, and also the resolution is not optimal. The simple AD structure would realize a low production cost, compared with the complicated dynode manufacture process, and its symmetric round shape brings a uniform response as well.

At first, the $20 \mathrm{~cm}$ HPD with a normal QE (22\% at peak), which is the proposed size for the outer veto layer in Hyper-K, was developed for the proof test. The high-QE $50 \mathrm{~cm}$ HPD is also being developed.

\section{$2.250 \mathrm{~cm}$ High-QE Super-Kamiokande Photomultiplier Tube}

The $50 \mathrm{~cm}$ Super-K PMT uses a Venetian blind dynode with eleven stages for the electron amplification and has been working since 1996 in Super-K. Therefore, these characteristics are well known and reliable.

Figure [1] shows the measured QE as a function of wavelength, where solid lines of $50 \mathrm{~cm}$ high-QE Super-K PMT samples are compared with a dotted line of the typical Super-K PMT. The new PMT shows 30\% QE at peak, which is improved from 22\% QE used in Super-K.

\section{$2.350 \mathrm{~cm}$ High-QE Box-and-Line Photomultiplier Tube}

The high-QE Box\&Line PMT was improved from the Super-K PMT, and a $43 \mathrm{~cm}$ PMT with a box-and-line dynode (Hamamatsu R7250) for the KamLAND experiment. Because of the optimizations of the bulb shape and dynodes with a wide acceptance and focusing electrode, the active area reaches almost full of $50 \mathrm{~cm}$ diameter area. The box and line dynode realizes a good resolution by a uniform amplification path due to the line focused dynode, compared with the Venetian blind dynode. The box-shape first dynode also brings the better collection efficiency (CE) of photoelectron with its wide acceptance, resulted in $95 \%(85 \%)$ for an area of $50 \mathrm{~cm}(46 \mathrm{~cm})$ diameter, compared to $67 \%(61 \%)$ in the Super-K PMT. 


\section{Test in a 200 ton Water Cherenkov Detector}

\subsection{Setup}

A 200 ton water tank at Kamioka, Japan, which is developed for the EGADS (Evaluating Gadolinium's Action on Detector Systems) experiment [四], is utilized for the proof test. The goal of the proof test on new photodetectors is to evaluate performance and to confirm there is no problem in detection of trouble for a few years operation. The tank is designed to hold 240 photodetectors of the conventional Super-K PMTs, some of which were replarced with eight $20 \mathrm{~cm}$ HPDs and five $50 \mathrm{~cm}$ high-QE Super-K PMTs in 2013 summer. The signal is transported from the tank by 70 meters RG-58/U coaxial cable, then the charge and time were recorded by an electronics module developed for Super-K, which was used until 2008. The first phase of test was started from September 2013, and a continuous operation was performed from February 2014 to May 2014.

Three $50 \mathrm{~cm}$ high-QE Box\&Line PMTs were also placed into the tank in 2014 summer for the second phase test. Because one of the $20 \mathrm{~cm}$ HPDs had a trouble in the high voltage module equipped inside, and another one got a high noise rate over $100 \mathrm{kHz}$, these two HPDs were replaced with new HPDs with improved high voltage power supply to fix the malfunction found. Then the next phase of test started from September 2014. A continuous run is started from November after the calibration. The $50 \mathrm{~cm}$ high-QE HPDs will also join in early 2016 after a preamplifier becomes ready.

Figure 7 indicates the positions of $20 \mathrm{~cm}$ HPDs and $50 \mathrm{~cm}$ high-QE PMTs in a development view of the tank, with $50 \mathrm{~cm}$ high-QE Box\&Line PMTs added later in 2014.

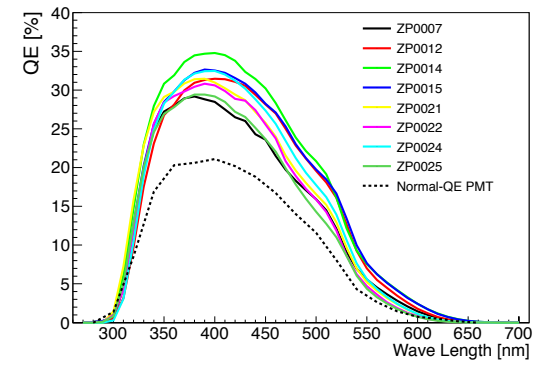

Figure 3: QE spectra of several $50 \mathrm{~cm}$ high-QE Super-K PMTs compared with the conventional Super-K PMT in dotted line.

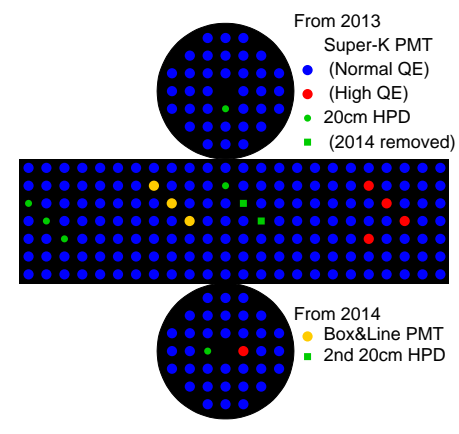

Figure 4: Development view of the 200 ton water tank.

\subsection{Performance of Single Photoelectron Detection}

The charge and time resolutions with single photoelectron signal were measured for all photodetectors simultaneously, by injecting a pulsed light at the center of the tank. For several HPDs, resolutions are low because an $\mathrm{AD}$ gain is differently set at half level from others due to a low maximum gain by an individual difference during an optimization of the production in the development, which will be solved in a future production.

The charge resolution for a single photoelectron signal is shown for all photodetectors in Figure [1. The Box\&Line PMTs show a charge resolution of $40-45 \%$ in $\sigma$, while it is around $30-35 \%$ 
in case of the $20 \mathrm{~cm}$ HPDs except for a low gain group whose performance is limited by the preamplifier.

The time resolution, defined as full width at half maximum (FWHM), is also shown in Figure 6. The time is measured at fixed hit thresholds of 0.25 photoelectrons for PMTs (corresponding to $1 \mathrm{mV}$ ) or 0.5 photoelectrons for HPDs $(4 \mathrm{mV})$, then the time walk is corrected for each photodetector. Resulted resolution is $5.1 \mathrm{nsec}$ in average of three Box\&Line PMTs, which is currently superior to the $20 \mathrm{~cm}$ HPD's due to a large noise and slow response by the preamplifier.

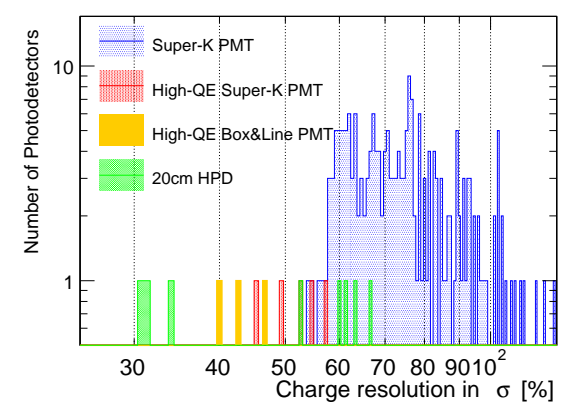

Figure 5: Charge resolution in sigma Figure 6: Time resolution (FWHM) at sinat single photoelectron measured in the gle photoelectron measured in the 200 ton 200 ton water tank at Kamioka mine.

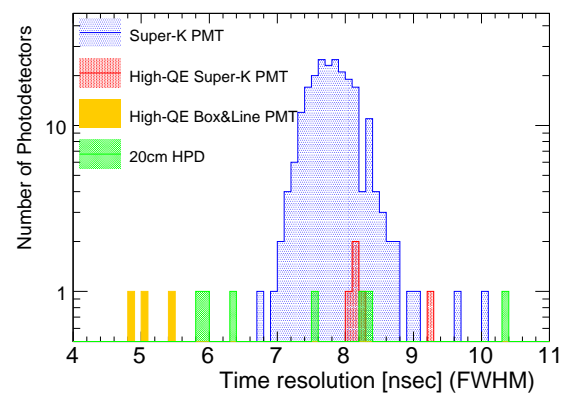

water tank at Kamioka mine.

\subsection{Dark Noise Rate}

A low noise hit rate in dark (dark rate) is essential to take the low energy events efficiently by these hit coincidence in Hyper-K. The dark rate at 0.25 photoelectron threshold $(1 \mathrm{mV})$ is $4.2 \mathrm{kHz}$ in Super-K, and generally its stabilization requires a long period of the order of years. After the initial rapid decrease, the dark rate was measured by taking average for eight months in each photodetector as shown in Figure $\mathbb{Z}$, where the hit threshold is same as used in the timing measurement.

The HPDs show a rate around $1 \mathrm{kHz}$, except for one failure HPD with high rate. The highQE Super-K PMTs show dark rate of $8.4 \mathrm{kHz}$ in average. It is high because of the high QE photocathode. The Box\&Line PMTs for the proof test are in initial trial production stage and indicate noisy rate around $20 \mathrm{kHz}$, but the second version of the Box\&Line PMT with dark rate and after pulse reduction achieved a dark rate comparable to that of the high-QE Super-K PMT (about $10 \mathrm{kHz}$ in room).

\subsection{Stability}

\subsubsection{Stability of Gain}

The stability of gain has been monitored by observing $1 \mathrm{~Hz}$ pulsed light from an optical diffuser ball in the tank. Figure 8 shows a one-month stability of the $20 \mathrm{~cm}$ HPDs' gain in 2014 April, where the peaks are normalized to 1 at the first point. The single photoelectron peak in the charge distribution was monitored and its RMS (root mean square) resulted in a range between 0.5 and $2 \%$ relatively. 


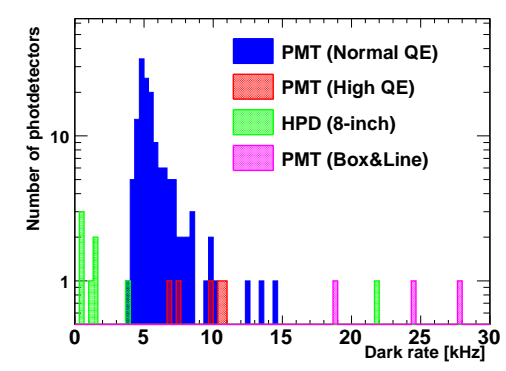

Figure 7: Dark rate distribution in the 200 ton water tank by eight-month average.

From November 2014, the light peak monitor was restarted with newly added three high-QE Box\&Line PMTs. Figure $\mathbb{Q}$ shows a result of peak monitoring of five high-QE Super-K PMTs and three high-QE Box\&Line PMTs for a signal corresponding to few tens of photoelectrons. The data from each photodetector is corrected by the average size of signal of other Super-K PMTs with normal $\mathrm{QE}$, in order to compensate change of the water transparency and the light intensity. The RMS of variation of the high-QE Super-K PMTs is $0.4-0.9 \%$, while that is $1.0-1.6 \%$ in the high-QE Box\&Line PMTs. The signal size from the Box\&Line PMTs seems to change slowly, and its gradual change is a true gain shift confirmed by another gain evaluation. In Hyper-K it is acceptable, because this kind of change is also seen in Super-K, and can be calibrated by $1 \mathrm{~Hz}$ light monitoring as has been done in Super-K.

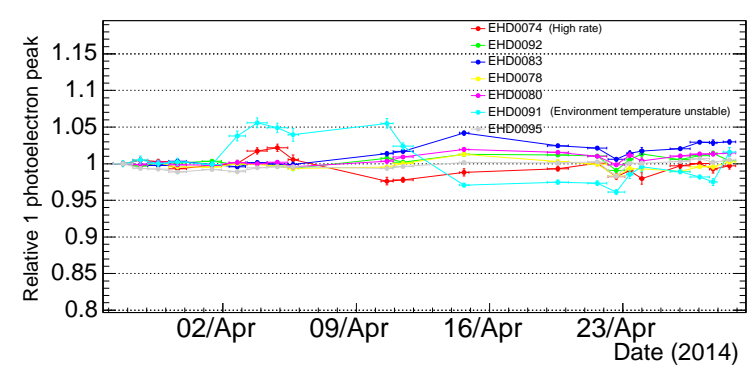

Figure 8: Individual HPD gain monitored by the single photoelectron peak without any gain correction.

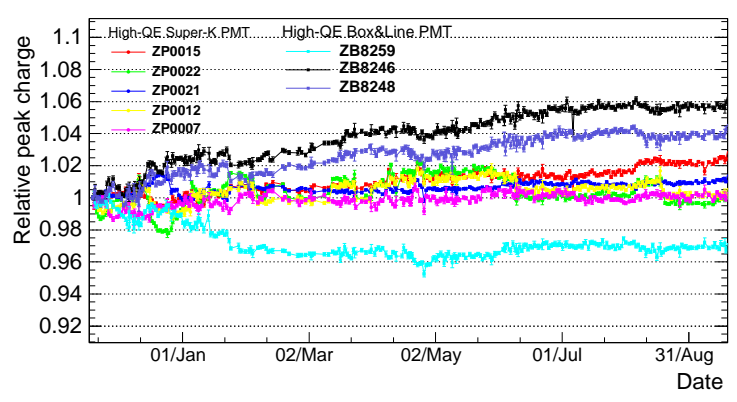

Figure 9: Individual high-QE PMT output monitored by the light peak, which is corrected with normal QE Super-K PMT average at each monitoring point.

\subsubsection{Stability of Dark Noise Rate}

The stability of the dark rate was also monitored by a $200 \mathrm{~Hz}$ periodic trigger for 5 minutes every day. Figures $\square$ and $\square$ show the dark rate of new photodetectors measured for ten months from 2014 to 2015. Because a high AD bias had been set in one HPD (EHD0083) near to the break down voltage, the rate had been temporarily high but was recovered after the voltage was lowered. In addition, one of the newly installed HPDs (EHD0104) is unstable and getting high rate, which has a low AD gain limited by a low breakdown voltage. Among five and three high-QE PMTs of each new type, only one Box\&Line PMT got high above $30 \mathrm{kHz}$ (Figure $\mathrm{W}$ ). 
Currently in Super-K, the fraction of PMTs with high dark rate among a large number of photosensors is at $1 \%$ level. Though it is still under trial optimization of the production, we try to suppress and avoid such a failure like high rate or flashing as much as possible in Hyper-K mass production, with pretest before the installation into Hyper-K.
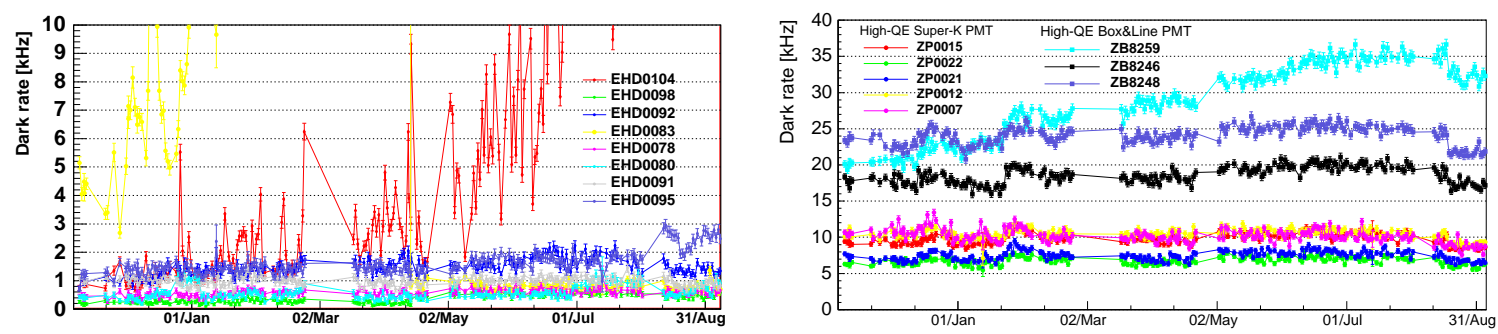

Figure 10: Individual HPD dark rate measured to Figure 11: Individual high-QE PMT dark rate meamonitor its stability. One HPD got high rate as shown sured to monitor its stability. Top three lines show the by a red line (EHD0104). Another HPD in yellow Box\&Line PMTs, and the dark rate for one photosen(EHD0083) showed high rate because the AD bias sor (ZB8259) is getting a little high.

voltage was near to the break down voltage, and re-

covered after the voltage set is a little lowered.

\section{Summary and Prospect}

Two new $50 \mathrm{~cm}$ photodetectors, HPD and Box\&Line PMT, should be tested in the water Cherenkov detector to prove the feasibility for Hyper-K. The high performance and stable operation were confirmed on $20 \mathrm{~cm}$ HPDs and high-QE Box\&Line PMTs, to be usable in Hyper-K.

There were two HPDs and one Box\&Line PMTs getting high dark rate. A stable production should be established with minimizing the failures. The quality of about two hundreds of selected Hyper-K photodetectors will be evaluated in a Hyper-K prototype test from 2017. The test is planned with a similar water tank to demonstrate photodetectors, those cover and supporter, electronics for Hyper-K. Because one of the HPD high voltage modules got broken, the improved version was prepared and tested in the tank. Other solutions are also considered with alternative productions by other companies, or an external multi-channel power supply by developing a high voltage cable and connector for the HPD. The dark rate level of the Box\&Line PMTs was around $20 \mathrm{kHz}$, but the recent production shows a comparable level with the high-QE Super-K PMT.

The $50 \mathrm{~cm}$ high-QE HPD will also join in the proof test from early 2016. The proof test would allow us to establish the long term operation of new photodetectors, and to determine the best photodetector for Hyper-K.

\section{References}

[1] K. Abe et al., arXiv:1109.3262 [hep-ex] (2011).

[2] The Super-Kamiokande Collaboration, Nucl. Instrum. Meth. A501 (2003) 418.

[3] A. Suzuki et al., Nucl. Instrum. Meth. A329 (1993) 299.

[4] T. Mori for the Super-Kamiokande Collaboration, Nucl. Instrum. Meth. A732 (2013) 316. 